\title{
A DEVELOPMENT METHOD OF CUTTING FORCE COEFFICIENTS IN FACE MILLING PROCESS USING PARALLELOGRAM INSERT
}

\author{
Nhu-Tung Nguyen \\ HaUI Institute of Technology - HIT \\ Hanoi University of Industry \\ 298 Cau Dien str., Bac Tu Liem District, Hanoi, 100000 \\ tungnn@haui.edu.vn
}

\begin{abstract}
This paper presents a modeling method of cutting force and a combination approach of theory and experimental methods in the determination of cutting force coefficients in the face milling process using a parallelogram insert. By the theoretical method, the cutting forces were modeled by a mathematical function of cutting cutter geometry (Cutter diameter, the number of inserts, the insert nose radius, nsert cutting edge helix angle, etc.), cutting conditions (depth of cut, feed per flute, spindle speed, etc.), and cutting force coefficients (shear force coefficients, edge force coefficients). By the theoretical method, the average cutting forces in three directions (feed $-x$, normal $-y$, and axial $-z$ ) were modeled as the linear functions of feed per flute. By the experimental method, the average cutting forces in these three directions were also regressed as the linear functions of feed per flute with quite large determination coefficients $\left(R^{2}\right.$ were larger than $92 \%$ ). Then, the relationship of average cutting forces and feed per flute was used to determine all six cutting force coefficient components. The validation experiments were performed to verify the linear function of average cutting forces, to determine the cutting force coefficients, and to verify the cutting force models in the face milling process using a cutter with one parallelogram insert. The cutting force models were successfully verified by comparison of the shape and the values of predicted cutting forces and measured cutting forces. These proposed methods and models can be applied to determine the cutting force coefficients and predict the cutting force in the face milling process using a parallelogram insert and can be extended with other cutting types or other insert types.

Keywords: modeling, force models, cutting forces, force coefficients, average cutting forces, shear cutting coefficients, edge cutting coefficients, face milling, parallelogram insert, C45.
\end{abstract}

DOI: $10.21303 / 2461-4262.2021 .001890$

\section{Introduction}

Nowadays, milling is very popularly employed in computer numerical control (CNC) machines for metal material cutting operations. In the milling process, the cutting forces are the important parameter to predict the machining characteristics such as cutting power, vibrations, chatter, surface roughness, etc., and develop the machining processes with high effectiveness and low cost. The cutting force model plays a decisive role in accuracy when predicting the forces and other machining characteristics in machining processes, especially in milling processes.

Two modeling methods were generally used to model the cutting force in the machining process. In the first method, the cutting force was modeled by the regression model through experimental data. By using this method, the effect of the controllable factors such as cutting conditions, tool geometry, etc., on the cutting force was investigated. This method is easy to perform, and the cutting force model is also easy to model. However, this method was only applied in limited conditions, and the regression model changed with different conditions of experimental. Besides, this method was only applied separately for each pair of tool and workpiece, and the cutting force model changed when applying for different pairs of tool and workpiece. Several researchers applied this method in the investigation of cutting force in the face milling process of SKD61 Hard Steel [1], flat milling process of aerospace aluminum alloys [2], ball milling graded material [3], and so on. In the second method, the cutting force was modeled by the theoretical model through a mathematical function of tool geometry, tool, and workpiece material properties, cutting conditions, and so on. This method is a quite complex method in the modeling process. However, the modeling process was started from the analysis of the metal cutting principle that was the essence of cutting processes; so, this method had been widely applied in cutting processes such as turning, milling, drilling, etc. The reviews of this method are presented in the following. 
In milling processes, two model types were often applied to predict the cutting forces. In the first one, the cutting forces are proportional to the chip thickness and cutting force coefficients. In this model, the cutting force coefficients were expressed as the exponential functions of average chip thickness (exponential force coefficient model) [4,5]. Using this model, the instantaneous cutting force coefficients were predicted from the experimental data of average chip thickness, the instantaneous cutting force, and the average cutting force [6, 7]. This model is convenient for the analysis of dynamic cutting and stability lobes in the machining process. However, the edge forces were often neglected in the cutting force model.

In the second model, the cutting forces are often separated into the shear force and edge force and the average cutting force could be expressed by a linear function of feed rate. This linear function was used to determine three shear force coefficients and three edge force coefficients from the cutting test data of cutting force $[8,9]$. This model is easy to apply when the cutting forces can be measured in milling processes. So, it was applied to many types of milling tools such as flat end mill [9], ball-end mill [10], bull-end mill [11], and face milling [12], etc. In the face milling process, the cutting forces were modeled depending on the relationship of average cutting forces and feed per flute when milling using several types of the cutter inserts such as round cutter inserts [12], rectangular and convex triangular cutter inserts [13]. It seems that the cutting force models in face milling processes using the parallelogram cutter inserts have not been mentioned in the previous studies. While, nowadays, these cutter inserts are popularly using in the milling process both for rough milling and finish milling processes. Therefore, modeling of cutting forces in the milling process using the parallelogram cutter inserts are necessary to apply in the prediction of cutting forces and other machining characteristics of these milling processes.

This study was performed to model the cutting forces, to verify the relationship of average cutting forces and feed per flute, to determine the cutting force coefficients, and to verify the cutting force models in the milling process using the parallelogram cutter inserts.

\section{Materials and methods}

In face milling process using the parallelogram insert, the cutting process was the interactive process of cutter inserts and workpiece. During the relative movement of the cutter and the workpiece, the chip is formed from the geometrical intersection process of the cutter inserts and the workpiece. The chip geometry depends on the geometry of the workpiece, the geometry of the cutter inserts, and the relative position of the cutter inserts and workpiece. The geometrical model of face milling process was described in Fig. 1.

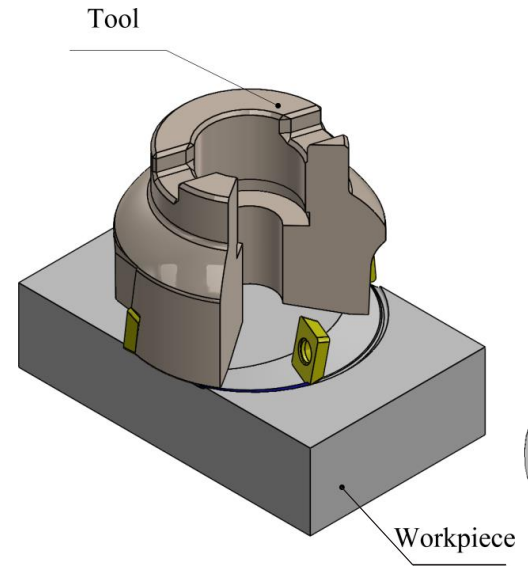

$a$

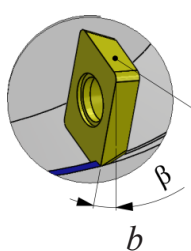

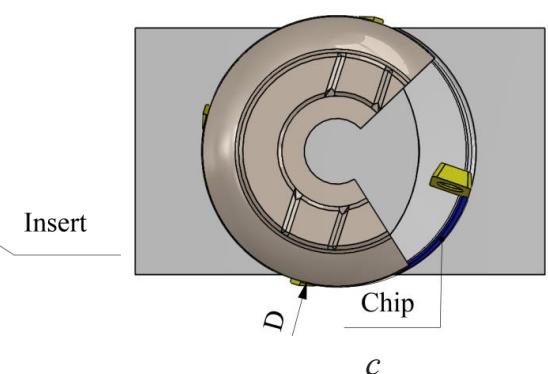

Fig. 1. Face milling process: $a$ - Tool and workpiece; $b$ - Parallelogram Insert; $c-$ Chip

In face milling process, the immersion position angle of each cutting edge is measured clockwise from the normal axis. Assuming that the bottom end of flute number one is designated as the reference immersion position angle $\left(\phi_{1}\right)$ and the bottom end point of the flute number $j$ is at 
an angle $\left(\phi_{j}\right)$, as shown in Fig. 2. So, the immersion position angle of the flute number $j\left(\phi_{j}\right)$ can be calculated by (1):

$$
\phi_{j}=\phi_{1}-(j-1) \phi_{p}, J=1 \sim N_{f},
$$

where $\phi_{p}$ is the cutter pitch angle that is the lag angle of two cutter inserts side by side as presented by (2):

$$
\phi_{p}=\frac{2 \pi}{N_{f}} .
$$

By the influence of the cutter helix angle, the radial lag angle $\psi$ at each axial depth of cut $z$ can be expressed in (3):

$$
\psi=\frac{2 \tan \beta}{D} z=\frac{\tan \beta}{R} z
$$

At an axial depth of cut $z$, the immersion angle is $\phi_{j}(z)$ of the flute number $j$ can be calculated by (4), as shown in Fig. 2.

$$
\phi_{j}(z)=\phi_{j}-\psi=\phi_{j}-\frac{\tan \beta}{R} z
$$

In case the depth of cut is smaller than the insert nose radius, the cutting process only occurs at part (1) of cutting edge. In case, the depth of cut is larger than the insert nose radius, the cutting process occurs at both part (1) and part (2) of the cutting edge as illustrated in Fig. 3.

In general cases, the cutting process occurs at both part (1) and part (2) of the cutting edge, the instantaneous chip thickness will be different in the different parts of the cutting edge. In part (1) of cutting edge, the instantaneous chip thickness is $h_{j 1}\left(\phi_{j}(z)\right)$ and in part (2) of cutting edge, the instantaneous chip thickness is $h_{j 2}\left(\phi_{j}(z)\right)$. In part (1) of cutting edge, the instantaneous chip thickness $h_{j 1}\left(\phi_{j}(z)\right)$ is determined by $(5)$ :

$$
h_{j 1}\left(\phi_{j}(z)\right)=f_{t} \cdot \sin \left(\phi_{j}(z)\right) \cdot \sin (\kappa(z))
$$

where $\kappa(z)$ is the axial immersion angle at $z$ axial cutting depth. And $\sin (\kappa(z))$ can be calculated by (6):

$$
\sin (\kappa(z))=\frac{d\left(\phi_{j}(z)\right.}{r\left(\phi_{j}(z)\right.}=\frac{\sqrt{r^{2}-(r-z)^{2}}}{r}, d=\sqrt{r^{2}-(r-z)^{2}} .
$$

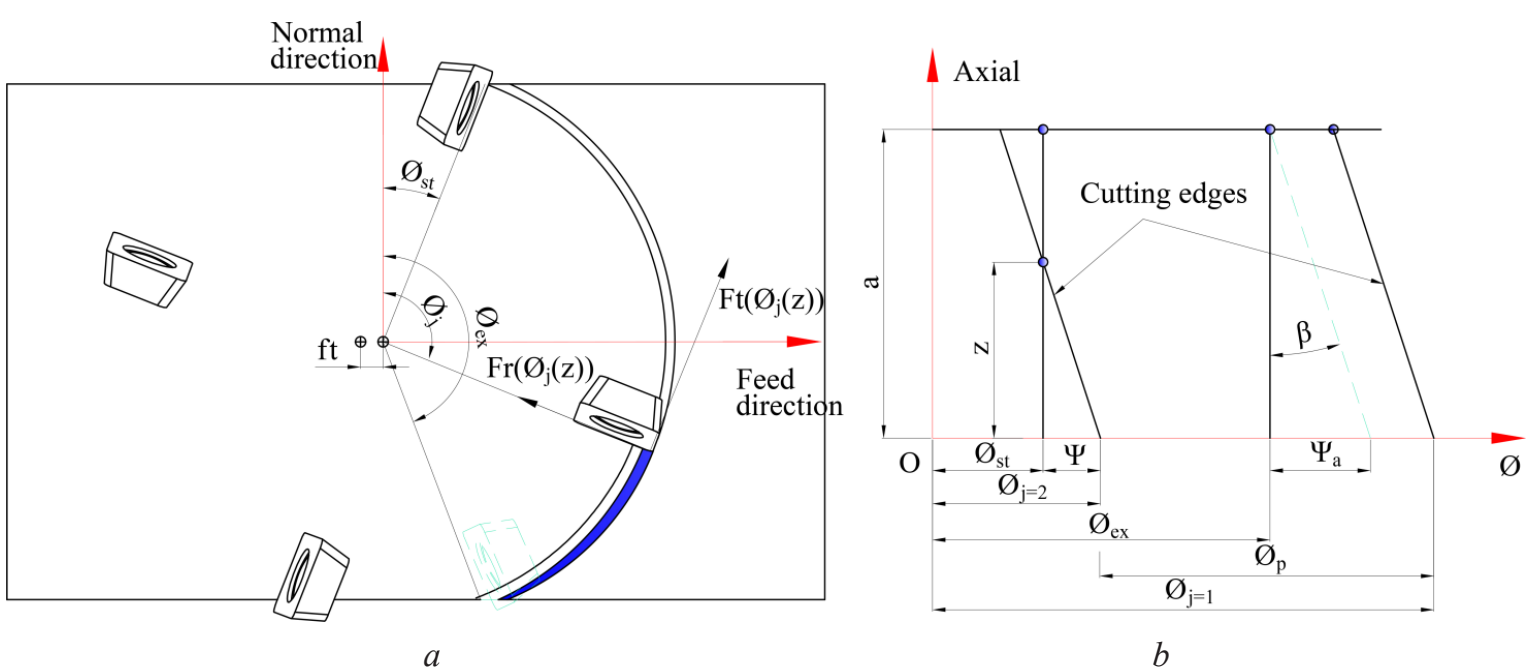

Fig. 2. The position angle of cutter inserts: $a$ - In feed and normal direction; $b-$ In axial direction 


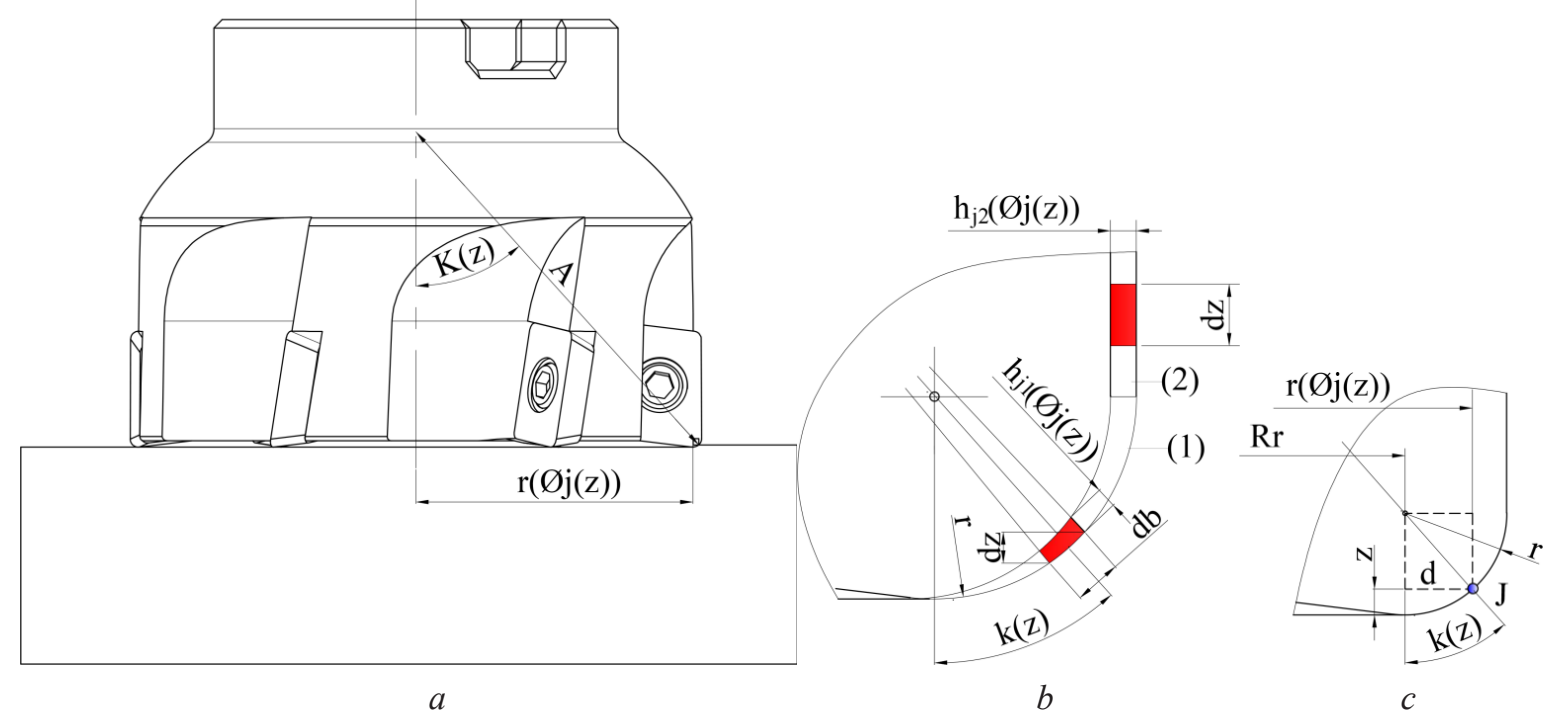

Fig. 3. Undeform chip thickness:

$a$ - Chip position; $b$ - Parts of chip; $c$ - Position of point $J$ in the chip

The immersion chip width (b) is calculated by (7):

$$
d b=\frac{d z}{\sin (\kappa(z))} .
$$

$r\left(\phi_{j}(z)\right)$ is the radius of a circle on $x y$ plane at an arbitrary point on the cutting edge. So, $r\left(\phi_{j}(z)\right)$ can be determined by (8):

$$
r\left(\phi_{j}(z)\right)=R_{r}+d=R_{r}+\sqrt{r^{2}-(r-z)^{2}} .
$$

The edge length of the cutting segment ds is obtained by the small variation of a vector, which is obtained from the center point to $a$ point $J$ on the cutting edge (Fig. 3), with respect to the small variation of rotation angle $\phi$. The location of point $J$ on the flute number $j$ is difined by a vector in cylindrical coordinates as described in Fig. 3 and can be calculated by (9):

$$
\vec{r}\left(\phi_{j}(z)\right)=r\left(\phi_{j}(z)\right) \cdot \sin \left(\phi_{j}(z)\right) \cdot \vec{i}+r\left(\phi_{j}(z)\right) \cdot \cos \left(\phi_{j}(z)\right) \cdot \vec{j}+z \cdot \vec{k}
$$

Where $z$ is the $z$ coordinate of point $J$, and, $d s$ can be derived by (10), (11):

$$
\begin{gathered}
d s=\mid \vec{r}\left(\phi_{j}(z) \mid=\sqrt{\left\{\left[r\left(\phi_{j}(z)\right) \cdot \sin \left(\phi_{j}(z)\right)\right]^{\prime}\right\}^{2}+\left\{\left[r\left(\phi_{j}(z)\right) \cdot \cos \left(\phi_{j}(z)\right)\right]^{\prime}\right\}^{2}+\left\{[z]^{\prime}\right\}^{2}},\right. \\
d s=d z \cdot \sqrt{\frac{(r-z)^{2}}{r^{2}-(r-z)^{2}}+\left[\frac{R_{r}+\sqrt{r^{2}-(r-z)^{2}}}{R}\right]^{2} \tan ^{2} \beta+1}=M \cdot d z,
\end{gathered}
$$

with

$$
M=\sqrt{\frac{(r-z)^{2}}{r^{2}-(r-z)^{2}}+\left[\frac{R_{r}+\sqrt{r^{2}-(r-z)^{2}}}{R}\right]^{2} \tan ^{2} \beta+1},
$$

So, in the part (1) of cutting edge, the tangential, radial, and axial forces acting on a differential flute element can be expressed as in (13): 


$$
\left\{\begin{array}{l}
d F_{t 1, j}(\phi, z)=K_{t e} \cdot d s\left(\phi_{j}(z)\right)+K_{t c} \cdot h_{j}\left(\phi_{j}(z)\right) \cdot d b \\
d F_{r 1, j}(\phi, z)=K_{r e} \cdot d s\left(\phi_{j}(z)\right)+K_{r c} \cdot h_{j}\left(\phi_{j}(z)\right) \cdot d b \\
d F_{a 1, j}(\phi, z)=K_{a e} \cdot d s\left(\phi_{j}(z)\right)+K_{a c} \cdot h_{j}\left(\phi_{j}(z)\right) \cdot d b
\end{array}\right.
$$

where $K_{t c}, K_{r c}, K_{a c}, K_{t e}, K_{r e}$ are the cutting force coefficients (The values of cutting force coefficients depend on the interaction mechanical of cutter and workpiece). Replacing (5), (7), and (11) into (13), the the tangential, radial, and axial forces can be calculated by (14):

$$
\left\{\begin{array}{l}
d F_{t 1, j}(\phi, z)=\left[K_{t e} \cdot M+K_{t c} \cdot f_{t} \cdot \sin \left(\phi_{j}(z)\right)\right] d z \\
d F_{r 1, j}(\phi, z)=\left[K_{r e} \cdot M+K_{r c} \cdot f_{t} \cdot \sin \left(\phi_{j}(z)\right)\right] d z \\
d F_{a 1, j}(\phi, z)=\left[K_{a e} \cdot M+K_{a c} \cdot f_{t} \cdot \sin \left(\phi_{j}(z)\right)\right] d z
\end{array}\right.
$$

The cutting forces are often measured in three directions that including feed ( $f$ or $x)$, normal ( $n$ or $y$ ), and axial $(z)$ directions. So, using the transformation method, the elemental cutting forces in feed, normal, and axial force are calculated by (15), (16) as shown in Fig. 4:

$$
\left\{\begin{array}{l}
d F_{f 1, j}(\phi, z) \\
d F_{n 1, j}(\phi, z) \\
d F_{z 1, j}(\phi, z)
\end{array}\right\}=\left[\begin{array}{ccc}
\cos \left(\phi_{j}(z)\right) & +\sin \left(\phi_{j}(z)\right) \cdot \sin (\kappa(z)) & +\sin \left(\phi_{j}(z)\right) \cdot \cos (\kappa(z)) \\
\sin \left(\phi_{j}(z)\right) & -\cos \left(\phi_{j}(z)\right) \cdot \sin (\kappa(z)) & -\cos \left(\phi_{j}(z)\right) \cdot \cos (\kappa(z)) \\
0 & \cos (\kappa(z)) & -\sin (\kappa(z))
\end{array}\right]\left\{\begin{array}{l}
d F_{t 1, j}(\phi, z) \\
d F_{r 1, j}(\phi, z) \\
d F_{a 1, j}(\phi, z)
\end{array}\right\},(15)
$$

so,

$$
\Rightarrow\left\{\begin{array}{l}
d F_{f 1, j}(\phi, z)=\cos \left(\phi_{j}-\frac{\tan \beta}{R} z\right) \cdot d F_{t 1, j}(\phi, z)+\sin \left(\phi_{j}-\frac{\tan \beta}{R}\right) \cdot \sin (\kappa(z)) \cdot d F_{r 1, j}(\phi, z)+ \\
+\sin \left(\phi_{j}-\frac{\tan \beta}{R} z\right) \cdot \cos (\kappa(z)) \cdot d F_{a 1, j}(\phi, z) ; \\
d F_{n 1, j}(\phi, z)=\sin \left(\phi_{j}-\frac{\tan \beta}{R} z\right) \cdot d F_{t 1, j}(\phi, z)-\cos \left(\phi_{j}-\frac{\tan \beta}{R} z\right) \cdot \sin (\kappa(z)) \cdot d F_{r 1, j}(\phi, z)- \\
-\cos \left(\phi_{j}-\frac{\tan \beta}{R} z\right) \cdot \cos (\kappa(z)) \cdot d F_{a 1, j}(\phi, z) ; \\
d F_{z 1, j}(\phi, z)=\cos (\kappa(z)) \cdot d F_{r 1, j}(\phi, z)-\sin (\kappa(z)) \cdot d F_{a 1, j}(\phi, z) .
\end{array}\right.
$$

In part (2) of cutting edge, the instantaneous chip thickness $h_{j 2}\left(\phi_{j}(z)\right)$ is determined by (17):

$$
h_{j 2}\left(\phi_{j}(z)\right)=f_{t} \cdot \sin \left(\phi_{j}(z)\right) \text {. }
$$

So, in this part, the tangential, radial, and axial cutting forces acting on a differential flute element can be expressed as in (18):

$$
\left\{\begin{array}{l}
\bar{F}_{f 2}=\frac{N_{f}}{2 \pi} \int_{\phi_{s t}+_{a 1}}^{\phi_{e x}+_{a 2}}\left(\int_{z_{j, 2}}^{z_{j, 3}} d F_{f 2, j}(\phi(z))\right) \mathrm{d} \phi ; \\
\bar{F}_{n 2}=\frac{N_{f}}{2 \pi} \int_{\phi_{s t}+_{a 1}}^{\phi_{e x}+_{a 2}}\left(\int_{z_{j, 2}}^{z_{j, 3}} d F_{n 2, j}(\phi(z))\right) \mathrm{d} \phi ; \\
\bar{F}_{a 2}=\frac{N_{f}}{2 \pi} \int_{\phi_{s t}+_{a 1}}^{\phi_{e x}+_{a 2}}\left(\int_{z_{j, 2}}^{z_{j, 3}} d F_{a 2, j}(\phi(z))\right) \mathrm{d} \phi
\end{array}\right.
$$

Using the transformation method, the feed, normal, and axial cutting forces are calculated by (19), (20): 


$$
\begin{gathered}
\left\{\begin{array}{l}
D F_{f 2, j}(\phi, z) \\
d F_{n 2, j}(\phi, z) \\
d F_{z 2, j}(\phi, z)
\end{array}\right\}=\left[\begin{array}{ccc}
-\cos \left(\phi_{j}(z)\right) & -\sin \left(\phi_{j}(z)\right) & 0 \\
\sin \left(\phi_{j}(z)\right) & -\cos \left(\phi_{j}(z)\right) & 0 \\
0 & 0 & 1
\end{array}\right]\left[\begin{array}{l}
d F_{t 2, j}(\phi, z) \\
d F_{r 2, j}(\phi, z) \\
d F_{a 2, j}(\phi, z)
\end{array}\right\},
\end{gathered}
$$

So, in general cases, the cutting forces occur at both part (1) and part (2) of the cutting edge as shown in Fig. 5.

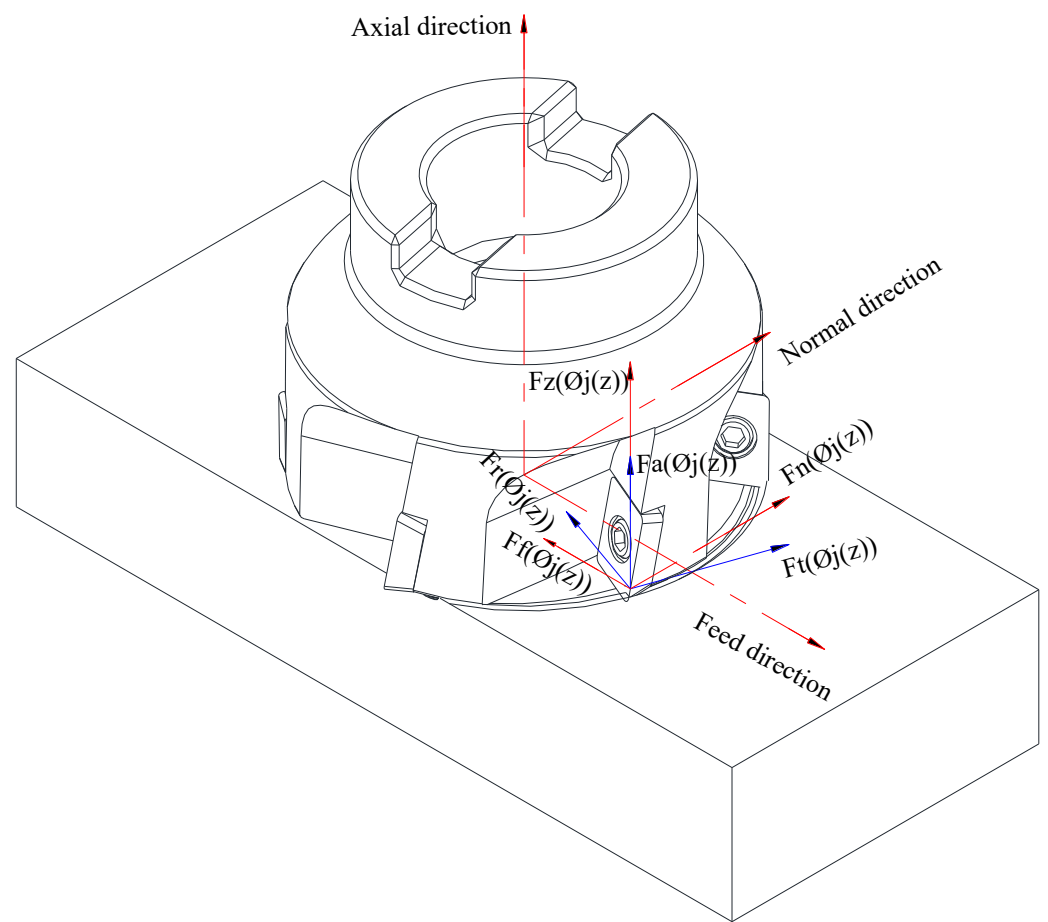

Fig. 4. Cutting forces in cutting edge 1

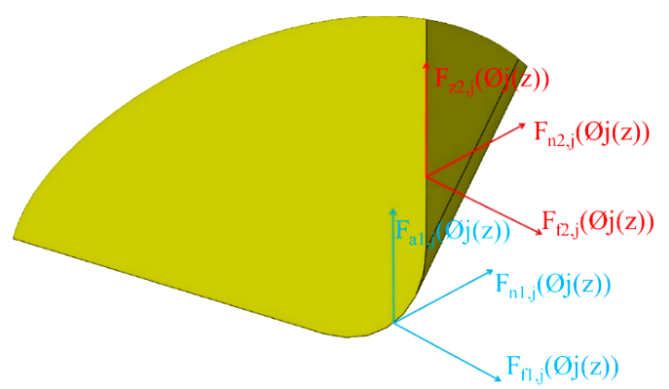

Fig. 5. Cutting force in two parts of cutting edge

To determine the total cutting force, the differential cutting forces are integrated analytically along the in-cut portion of the cutting edge of flute $j$. So, the total cutting forces in each cutting edge can be calculated by (21):

$$
F_{q}\left(\phi_{j}(z)\right)=F_{q 1}\left(\phi_{j}, z\right)+F_{q 2}\left(\phi_{j}, z\right)=\int_{z_{1\left(\phi_{j}\right)}}^{z_{z\left(\phi_{j}\right)}} \mathrm{d} F_{q 1}\left(\phi_{j}, z\right)+\int_{z_{2\left(\phi_{j}\right)}}^{z_{3\left(\phi_{j}\right)}} \mathrm{d} F_{q 2}\left(\phi_{j}, z\right) \text { with } q=f, n, a .
$$


In cases, there are more than one flute executing the cutting processes simultaneously, the total cutting forces in the feed, normal, and axial directions can be calculated by (22):

$$
F_{q}\left(\phi_{j}\right)=\sum_{j=1}^{N_{f}} F_{q, j}\left(\phi_{j}, z\right) .
$$

In the face milling process, the average cutting forces of $N_{f}$ flutes in feed, normal, and axial directions per revolution can be calculated by (23):

$$
\left\{\begin{array}{l}
\bar{F}_{f}=\bar{F}_{f 1}+\bar{F}_{f 2} ; \\
\bar{F}_{n}=\bar{F}_{n 1}+\bar{F}_{n 2} ; \\
\bar{F}_{z}=\bar{F}_{z 1}+\bar{F}_{z 2} .
\end{array}\right.
$$

In the part (1) of the cutting edge, the average cutting forces of $N_{f}$ flutes in feed, normal, and axial directions, per revolution can be expressed by (24):

$$
\left\{\begin{array}{l}
\bar{F}_{f 1}=\frac{N_{f}}{2 \pi} \int_{\phi_{s t}}^{\phi_{e x}+\Psi_{a 1}}\left(\int_{z_{j, 1}}^{z_{j, 2}} d F_{f 1, j}(\phi(z))\right) \mathrm{d} \phi ; \\
\bar{F}_{n 1}=\frac{N_{f}}{2 \pi} \int_{\phi_{s t}}^{\phi_{e x}+\Psi_{a 1}}\left(\int_{z_{j, 1}}^{z_{j, 2}} d F_{n 1, j}(\phi(z))\right) \mathrm{d} \phi ; \\
\bar{F}_{a 1}=\frac{N_{f}}{2 \pi} \int_{\phi_{s t}}^{\phi_{e x}+\Psi_{a 1}}\left(\int_{z_{j, 1}}^{z_{j, 2}} d F_{a 1, j}(\phi(z))\right) \mathrm{d} \phi .
\end{array}\right.
$$

In the part (2) of the cutting edge, the average cutting forces of $N_{f}$ flutes in feed, normal, and axial directions, per revolution can be expressed by (25):

$$
\left\{\begin{array}{l}
\bar{F}_{f 2}=\frac{N_{f}}{2 \pi} \int_{\phi_{s t}+_{a 1}}^{\phi_{e x}+_{a 2}}\left(\int_{z_{j, 2}}^{z_{j, 3}} d F_{f 2, j}(\phi(z))\right) \mathrm{d} \phi ; \\
\bar{F}_{n 2}=\frac{N_{f}}{2 \pi} \int_{\phi_{s t}+_{a 1}}^{\phi_{e x}+_{a 2}}\left(\int_{z_{j, 2}}^{z_{j, 3}} d F_{n 2, j}(\phi(z))\right) \mathrm{d} \phi ; \\
\bar{F}_{a 2}=\frac{N_{f}}{2 \pi} \int_{\phi_{s t}+_{a 1}}^{\phi_{e x}+_{a 2}}\left(\int_{z_{j, 2}}^{z_{j, 3}} d F_{a 2, j}(\phi(z))\right) \mathrm{d} \phi .
\end{array}\right.
$$

Where $\phi_{s t},\left(\phi_{s t}+\psi_{a 1}\right), \phi_{e x},\left(\phi_{e x}+\psi_{a 2}\right)$ are cutting boundary conditions.

Set the constants $C_{i}$ by from (26) to (46):

$$
\begin{gathered}
C_{1}=-\frac{N_{f}}{4 \pi}\left[\begin{array}{l}
\left.\int_{\phi_{s t}}^{\phi_{s t}+\psi_{a 1}}\left(\int_{0}^{\frac{R}{\tan \beta}\left(\phi_{j}-\phi_{s t}\right)}\left(\sin 2\left(\phi_{j}-\frac{\tan \beta}{R} z\right)\right) \mathrm{d} z\right) d \phi+\right] \\
+\int_{\phi_{s t}+\psi_{a 1}}^{\phi_{e x}}\left(\int_{0}^{r}\left(\sin 2\left(\phi_{j}-\frac{\tan \beta}{R} z\right)\right) \mathrm{d} z\right) \mathrm{d} \phi+ \\
+\int_{\phi_{e x}}^{\phi_{e x}+\psi_{a 1}}\left(\int_{\frac{R}{\tan \beta}\left(\phi_{j}-\phi_{e x}\right)}^{r}\left(\sin 2\left(\phi_{j}-\frac{\tan \beta}{R} z\right)\right) \mathrm{d} z\right) \mathrm{d} \phi
\end{array}\right] \\
C_{2}=-\frac{N_{f}}{2 \pi}\left[\begin{array}{l}
\int_{\phi_{s t}}^{\phi_{s t}+\psi_{a 1}}\left(\int_{0}^{\frac{R}{\tan \beta}\left(\phi_{j}-\phi_{s t}\right)}\left(\cos \left(\phi_{j}-\frac{\tan \beta}{R} z\right) \cdot M\right) \mathrm{d} z\right) \mathrm{d} \phi+ \\
+\int_{\phi_{s t}+\psi_{\psi a 1}}^{\phi_{e x}}\left(\int_{0}^{r}\left(\cos \left(\phi_{j}-\frac{\tan \beta}{R} z\right) \cdot M\right) \mathrm{d} z\right) \mathrm{d} \phi+ \\
+\int_{\phi_{e x}}^{\phi_{e x}+\psi_{a 1}}\left(\int_{\frac{R}{\tan \beta}\left(\phi_{j}-\phi_{e x}\right)}^{r}\left(\cos \left(\phi_{j}-\frac{\tan \beta}{R} z\right) \cdot M\right) \mathrm{d} z\right) \mathrm{d} \phi
\end{array}\right],
\end{gathered}
$$




$$
\begin{aligned}
& C_{3}=-\frac{N_{f}}{4 \pi}\left[\begin{array}{l}
\left.\int_{\phi_{s t}}^{\phi_{s t}+\psi_{a 1}}\left(\int_{0}^{\frac{R}{\tan \beta}\left(\phi_{j}-\phi_{s t}\right)} \sin (K z) \cdot\left(1-\cos 2\left(\phi_{j}-\frac{\tan \beta}{R} z\right)\right) \mathrm{d} z\right) \mathrm{d} \phi+\right] \\
+\int_{\phi_{s t}+\psi_{a 1}}^{\phi_{e x}}\left(\int_{0}^{r} \sin (K z) \cdot\left(1-\cos 2\left(\phi_{j}-\frac{\tan \beta}{R} z\right)\right) \mathrm{d} z\right) \mathrm{d} \phi+ \\
+\int_{\phi_{e x}}^{\phi_{e x}+\psi_{a 1}}\left(\int_{\frac{R}{\tan \beta}\left(\phi_{j}-\phi_{e x}\right)}^{r} \sin (K z) \cdot\left(1-\cos 2\left(\phi_{j}-\frac{\tan \beta}{R} z\right)\right) \mathrm{d} z\right) \mathrm{d} \phi
\end{array}\right], \\
& C_{4}=-\frac{N_{f}}{2 \pi}\left[\begin{array}{l}
\int_{\phi_{s t}}^{\phi_{s t}+\psi_{a 1}}\left(\int_{0}^{\frac{R}{\tan \beta}\left(\phi_{j}-\phi_{s t}\right)}\left(\sin (K z) \cdot \sin \left(\phi_{j}-\frac{\tan \beta}{R} z\right) \cdot M\right) \mathrm{d} z\right) \mathrm{d} \phi+ \\
+\int_{\phi_{s t}+\psi_{a 1}}^{\phi_{e x}}\left(\int_{0}^{r}\left(\sin (K z) \cdot \sin \left(\phi_{j}-\frac{\tan \beta}{R} z\right) \cdot M\right) \mathrm{d} z\right) \mathrm{d} \phi+ \\
+\int_{\phi_{e x}}^{\phi_{e x}+\psi_{a 1}}\left(\int_{\frac{\tan \beta}{r}\left(\phi_{j}-\phi_{e x}\right)}^{r}\left(\sin (K z) \cdot \sin \left(\phi_{j}-\frac{\tan \beta}{R} z\right) \cdot M\right) \mathrm{d} z\right) \mathrm{d} \phi
\end{array}\right] \\
& C_{5}=-\frac{N_{f}}{4 \pi}\left[\begin{array}{l}
\int_{\phi_{s t}}^{\phi_{s t}+\psi_{a 1}}\left(\int_{0}^{\frac{R}{\tan \beta}\left(\phi_{j}-\phi_{s t}\right)}\left(\cos (K z) \cdot\left(1-\cos 2\left(\phi_{j}-\frac{\tan \beta}{R} z\right)\right)\right) \mathrm{d} z\right) \mathrm{d} \phi+ \\
+\int_{\phi_{s t}+\psi_{a 1}}^{\phi_{e x}}\left(\int_{0}^{r}\left(\cos (K z) \cdot\left(1-\cos 2\left(\phi_{j}-\frac{\tan \beta}{R} z\right)\right)\right) \mathrm{d} z\right) \mathrm{d} \phi- \\
+\int_{\phi_{e x}}^{\phi_{e x}+\psi_{a 1}}\left(\int_{\frac{R}{\tan \beta}\left(\phi_{j}-\phi_{e x}\right)}^{r}\left(\cos (K z) \cdot\left(1-\cos 2\left(\phi_{j}-\frac{\tan \beta}{R} z\right)\right)\right) \mathrm{d} z\right) \mathrm{d} \phi
\end{array}\right], \\
& C_{6}=-\frac{N_{f}}{2 \pi}\left[\begin{array}{l}
\left.\int_{\phi_{s t}}^{\phi_{s t}+\psi_{a 1}}\left(\int_{0}^{\frac{R}{\tan \beta}\left(\phi_{j}-\phi_{s t}\right)}\left(\cos (K z) \cdot \sin \left(\phi_{j}-\frac{\tan \beta}{R} z\right) \cdot M\right) \mathrm{d} z\right) \mathrm{d} \phi+\right] \\
+\int_{\phi_{s t}+\psi_{a 1}}^{\phi_{e x}}\left(\int_{0}^{r}\left(\cos (K z) \cdot \sin \left(\phi_{j}-\frac{\tan \beta}{R} z\right) \cdot M\right) \mathrm{d} z\right) \mathrm{d} \phi+ \\
+\int_{\phi_{e x}}^{\phi_{e x}+\psi_{a 1}}\left(\int_{\frac{R}{\tan \beta}\left(\phi_{j}-\phi_{e x}\right)}^{r}\left(\cos (K z) \cdot \sin \left(\phi_{j}-\frac{\tan \beta}{R} z\right) \cdot M\right) \mathrm{d} z\right) \mathrm{d} \phi
\end{array}\right], \\
& C_{7}=\frac{N_{f}}{4 \pi}\left[\begin{array}{l}
\int_{\phi_{s t}}^{\phi_{s t}+\psi_{a 1}}\left(\int_{0}^{\frac{R}{\tan \beta}\left(\phi_{j}-\phi_{s t}\right)}\left(1-\cos 2\left(\phi_{j}-\frac{\tan \beta}{R} z\right)\right) \mathrm{d} z\right) \mathrm{d} \phi+ \\
+\int_{\phi_{s t}+\psi_{a 1}}^{\phi_{e x}}\left(\int_{0}^{r}\left(1-\cos 2\left(\phi_{j}-\frac{\tan \beta}{R} z\right)\right) \mathrm{d} z\right) \mathrm{d} \phi+ \\
+\int_{\phi_{e x}}^{\phi_{e x}+\psi_{a} 1}\left(\int_{\frac{R}{\tan \beta}\left(\phi_{j}-\phi_{e x}\right)}^{r}\left(1-\cos 2\left(\phi_{j}-\frac{\tan \beta}{R} z\right)\right) \mathrm{d} z\right) \mathrm{d} \phi
\end{array}\right] \\
& C_{8}=\frac{N_{f}}{2 \pi}\left[\begin{array}{l}
\int_{\phi_{s t}}^{\phi_{s t}+\psi_{a 1}}\left(\int_{0}^{\frac{R}{\tan \beta}\left(\phi_{j}-\phi_{s t}\right)}\left(\sin \left(\phi_{j}-\frac{\tan \beta}{R} z\right)\right) \cdot M \mathrm{~d} z\right) \mathrm{d} \phi+ \\
+\int_{\phi_{s t}+\psi_{a 1}}^{\phi_{e x}}\left(\int_{0}^{r}\left(\sin \left(\phi_{j}-\frac{\tan \beta}{R} z\right)\right) \cdot M \mathrm{~d} z\right) \mathrm{d} \phi+ \\
+\int_{\phi_{e x}}^{\phi_{e x}+\psi_{a 1}}\left(\int_{\frac{R}{\tan \beta}\left(\phi_{j}-\phi_{e x}\right)}^{r}\left(\sin \left(\phi_{j}-\frac{\tan \beta}{R} z\right)\right) \cdot M \mathrm{~d} z\right) \mathrm{d} \phi
\end{array}\right],
\end{aligned}
$$




$$
\begin{aligned}
& C_{9}=-\frac{N_{f}}{4 \pi}\left[\begin{array}{l}
\int_{\phi_{s t}}^{\phi_{s t}+\psi_{a 1}}\left(\int_{0}^{\frac{R}{\tan \beta}\left(\phi_{j}-\phi_{s t}\right)}\left(\sin (K z) \cdot \sin 2\left(\phi_{j}-\frac{\tan \beta}{R} z\right)\right) \mathrm{d} z\right) \mathrm{d} \phi+ \\
+\int_{\phi_{s t}+\psi_{a 1}}^{\phi_{e x}}\left(\int_{0}^{r}\left(\sin (K z) \cdot \sin 2\left(\phi_{j}-\frac{\tan \beta}{R} z\right)\right) \mathrm{d} z\right) \mathrm{d} \phi+ \\
+\int_{\phi_{e x}}^{\phi_{e x}+\psi_{a 1}}\left(\int_{\frac{R}{\tan \beta}\left(\phi_{j}-\phi_{e x}\right)}^{r}\left(\sin (K z) \cdot \sin 2\left(\phi_{j}-\frac{\tan \beta}{R} z\right)\right) \mathrm{d} z\right) \mathrm{d} \phi
\end{array}\right], \\
& C_{10}=-\frac{N_{f}}{2 \pi}\left[\begin{array}{l}
\int_{\phi_{s t}}^{\phi_{s t}+\psi_{a 1}}\left(\int_{0}^{\frac{R}{\tan \beta}\left(\phi_{j}-\phi_{s t}\right)}\left(\sin (K z) \cdot \cos \left(\phi_{j}-\frac{\tan \beta}{R} z\right) \cdot M\right) \mathrm{d} z\right) \mathrm{d} \phi+ \\
+\int_{\phi_{s t}+\psi_{a 1}}^{\phi_{e x}}\left(\int_{0}^{r}\left(\sin (K z) \cdot \cos \left(\phi_{j}-\frac{\tan \beta}{R} z\right) \cdot M\right) \mathrm{d} z\right) \mathrm{d} \phi+ \\
+\int_{\phi_{e x}}^{\phi_{e x}+\psi_{a 1}}\left(\int_{\frac{R}{\tan \beta}\left(\phi_{j}-\phi_{e x}\right)}^{r}\left(\sin (K z) \cdot \cos \left(\phi_{j}-\frac{\tan \beta}{R} z\right) \cdot M\right) \mathrm{d} z\right) \mathrm{d} \phi
\end{array}\right] \\
& C_{11}=-\frac{N_{f}}{4 \pi}\left[\begin{array}{l}
\int_{\phi_{s t}}^{\phi_{s t}+\psi_{a 1}}\left(\int_{0}^{\frac{R}{\tan \beta}\left(\phi_{j}-\phi_{s t}\right)}\left(\cos (K z) \cdot \sin 2\left(\phi_{j}-\frac{\tan \beta}{R} z\right)\right) \mathrm{d} z\right) \mathrm{d} \phi+ \\
+\int_{\phi_{s t}+\psi_{a 1}}^{\phi_{e x}}\left(\int_{0}^{r}\left(\cos (K z) \cdot \sin 2\left(\phi_{j}-\frac{\tan \beta}{R} z\right)\right) \mathrm{d} z\right) \mathrm{d} \phi+ \\
+\int_{\phi_{e x}}^{\phi_{e x}+\psi_{a 1}}\left(\int_{\frac{R}{\tan \beta}\left(\phi_{j}-\phi_{e x}\right)}^{r}\left(\cos (K z) \cdot \sin 2\left(\phi_{j}-\frac{\tan \beta}{R} z\right)\right) \mathrm{d} z\right) \mathrm{d} \phi
\end{array}\right], \\
& C_{12}=-\frac{N_{f}}{2 \pi}\left[\begin{array}{l}
\int_{\phi_{s t}}^{\phi_{s t}+\psi_{a 1}}\left(\int_{0}^{\frac{R}{\tan \beta}\left(\phi_{j}-\phi_{s t}\right)}\left(\cos (K z) \cdot \cos \left(\phi_{j}-\frac{\tan \beta}{R} z\right) \cdot M\right) \mathrm{d} z\right) \mathrm{d} \phi+ \\
+\int_{\phi_{s t}+\psi_{a 1}}^{\phi_{e x}}\left(\int_{0}^{r}\left(\cos (K z) \cdot \cos \left(\phi_{j}-\frac{\tan \beta}{R} z\right) \cdot M\right) \mathrm{d} z\right) \mathrm{d} \phi+ \\
+\int_{\phi_{e x}}^{\phi_{e x}+\psi_{a 1}}\left(\int_{\frac{R}{\tan \beta}\left(\phi_{j}-\phi_{e x}\right)}^{r}\left(\cos (K z) \cdot \cos \left(\phi_{j}-\frac{\tan \beta}{R} z\right) \cdot M\right) \mathrm{d} z\right) \mathrm{d} \phi
\end{array}\right] \\
& C_{13}=\frac{N_{f}}{2 \pi}\left[\begin{array}{l}
\int_{\phi_{s t}}^{\phi_{s t}+\psi_{a 1}}\left(\int_{0}^{\frac{R}{\tan \beta}\left(\phi_{j}-\phi_{s t}\right)}\left(\cos (K z) \cdot \sin \left(\phi_{j}-\frac{\tan \beta}{R} z\right)\right) \mathrm{d} z\right) \mathrm{d} \phi+ \\
+\int_{\phi_{s t}+\psi_{a 1}}^{\phi_{e x}}\left(\int_{0}^{r}\left(\cos (K z) \cdot \sin \left(\phi_{j}-\frac{\tan \beta}{R} z\right)\right) \mathrm{d} z\right) \mathrm{d} \phi+ \\
+\int_{\phi_{e x}}^{\phi_{e x}+\psi_{a 1}}\left(\int_{\frac{R}{\tan \beta}\left(\phi_{j}-\phi_{e x}\right)}^{r}\left(\cos (K z) \cdot \sin \left(\phi_{j}-\frac{\tan \beta}{R} z\right)\right) \mathrm{d} z\right) \mathrm{d} \phi
\end{array}\right], \\
& C_{14}=\frac{N_{f}}{2 \pi}\left[\begin{array}{l}
\int_{\phi_{s t}}^{\phi_{s t}+\psi_{a 1}}\left(\int_{0}^{\frac{R}{\tan \beta}\left(\phi_{j}-\phi_{s t}\right)}(\cos (K z) \cdot M) \mathrm{d} z\right) \mathrm{d} \phi+ \\
+\int_{\phi_{s t}+\psi_{a 1}}^{\phi_{e x}}\left(\int_{0}^{r}(\cos (K z) \cdot M) \mathrm{d} z\right) \mathrm{d} \phi+ \\
+\int_{\phi_{e x}}^{\phi_{e x}+\psi_{a 1}}\left(\int_{0}^{r}(\cos (K z) \cdot M) \mathrm{d} z\right) \mathrm{d} \phi
\end{array}\right],
\end{aligned}
$$




$$
\begin{aligned}
& C_{15}=-\frac{N_{f}}{2 \pi}\left[\begin{array}{l}
\int_{\phi_{s t}}^{\phi_{s t}+\psi_{a 1}}\left(\int_{0}^{\frac{R}{\tan \beta}\left(\phi_{j}-\phi_{s t}\right)}\left(\sin \left((K z) \cdot \sin \left(\phi_{j}-\frac{\tan \beta}{R} z\right)\right) \mathrm{d} z\right) \mathrm{d} \phi+\right] \\
+\int_{\phi_{s t}+\psi_{a 1}}^{\phi_{e x}}\left(\int_{0}^{r}\left(\sin (K z) \cdot \sin \left(\phi_{j}-\frac{\tan \beta}{R} z\right)\right) \mathrm{d} z\right) \mathrm{d} \phi+ \\
+\int_{\phi_{e x}}^{\phi_{e x}+\psi_{a 1}}\left(\int_{\frac{R}{\tan \beta}\left(\phi_{j}-\phi_{e x}\right)}^{r}\left(\sin (K z) \cdot \sin \left(\phi_{j}-\frac{\tan \beta}{R} z\right)\right) \mathrm{d} z\right) \mathrm{d} \phi
\end{array}\right], \\
& C_{16}=-\frac{N_{f}}{2 \pi}\left[\begin{array}{l}
\int_{\phi_{s t}}^{\phi_{s t}+\psi_{a 1}}\left(\int_{0}^{\frac{R}{\tan \beta}\left(\phi_{j}-\phi_{s t}\right)}\left(\int_{0}^{\frac{R}{\tan \beta}\left(\phi_{j}-\phi_{s t}\right)}(\sin (K z) \cdot M) \mathrm{d} z\right) \mathrm{d} \phi\right)+ \\
+\int_{\phi_{s t}+\psi_{a 1}}^{\phi_{e x}}\left(\int_{0}^{r}(\sin (K z) \cdot M) \mathrm{d} z\right) \mathrm{d} \phi+ \\
+\int_{\phi_{e x}}^{\phi_{e x}+\psi_{a 1}}\left(\int_{\frac{R}{\tan \beta}\left(\phi_{j}-\phi_{e x}\right)}^{r}(\sin (K z) \cdot M) \mathrm{d} z\right) \mathrm{d} \phi
\end{array}\right], \\
& C_{17}=-\frac{N_{f}}{4 \pi}\left[\begin{array}{l}
\int_{\phi_{s t}+\psi_{a 1}}^{\phi_{s t}+\psi_{a 2}}\left(\int_{r}^{\frac{R}{\tan (\beta)} \cdot\left(\phi_{j}-\phi_{e x}\right)}\left(\sin 2\left(\phi_{j}-\frac{2 \tan \beta}{D} z\right)\right) \mathrm{d} z\right) \mathrm{d} \phi+ \\
+\int_{\phi_{s t}+\psi_{a 2}}^{\phi_{e x}+\psi_{a 1}}\left(\int_{r}^{a}\left(\sin 2\left(\phi_{j}-\frac{2 \tan \beta}{D} z\right)\right) \mathrm{d} z\right) \mathrm{d} \phi+ \\
+\int_{\phi_{e x}+\psi_{a 1}}^{\phi_{e x}+\psi_{a 2}}\left(\int_{\frac{R}{\tan (\beta)} \cdot\left(\phi_{j}-\phi_{e x}\right)}^{a}\left(\sin 2\left(\phi_{j}-\frac{2 \tan \beta}{D} z\right)\right) \mathrm{d} z\right) \mathrm{d} \phi
\end{array}\right], \\
& C_{18}=-\frac{N_{f}}{4 \pi}\left[\begin{array}{l}
\int_{\phi_{s t}+\psi_{a 1}}^{\phi_{s t}+\psi_{a 2}}\left(\int_{r}^{\frac{R}{\tan (\beta)} \cdot\left(\phi_{j}-\phi_{e x}\right)}\left(1-\cos 2\left(\phi_{j}-\frac{2 \tan \beta}{D} z\right)\right) \mathrm{d} z\right) \mathrm{d} \phi+ \\
+\int_{\phi_{s t}+\psi_{a 2}}^{\phi_{e x}+\psi_{a 1}}\left(\int_{r}^{a}\left(1-\cos 2\left(\phi_{j}-\frac{2 \tan \beta}{D} z\right)\right) \mathrm{d} z\right) \mathrm{d} \phi+ \\
+\int_{\phi_{e x}+\psi_{a 1}}^{\phi_{e x}+\psi_{a 2}}\left(\int_{\frac{R}{\tan (\beta)} \cdot\left(\phi_{j}-\phi_{e x}\right)}^{a}\left(1-\cos 2\left(\phi_{j}-\frac{2 \tan \beta}{D} z\right)\right) \mathrm{d} z\right) \mathrm{d} \phi
\end{array}\right], \\
& C_{19}=-\frac{N_{f}}{2 \pi}\left[\begin{array}{l}
\int_{\phi_{s t}+\psi_{a 1}}^{\phi_{s t}+\psi_{a 2}}\left(\int_{r}^{\frac{R}{\tan (\beta)} \cdot\left(\phi_{j}-\phi_{e x}\right)}\left(\cos \left(\phi_{j}-\frac{2 \tan \beta}{D} z\right)\right) \mathrm{d} z\right) \mathrm{d} \phi+ \\
+\int_{\phi_{s t}+\psi_{a 2}}^{\phi_{e x}+\psi_{a 1}}\left(\int_{r}^{a}\left(\cos \left(\phi_{j}-\frac{2 \tan \beta}{D} z\right)\right) \mathrm{d} z\right) \mathrm{d} \phi+ \\
+\int_{\phi_{e x}+\psi_{a 1}}^{\phi_{e x}+\psi_{a 2}}\left(\int_{\frac{R}{\tan (\beta)} \cdot\left(\phi_{j}-\phi_{e x}\right)}^{a}\left(\cos \left(\phi_{j}-\frac{2 \tan \beta}{D} z\right)\right) \mathrm{d} z\right) \mathrm{d} \phi
\end{array}\right], \\
& C_{20}=-\frac{N_{f}}{2 \pi}\left[\begin{array}{l}
\int_{\phi_{s t}+\psi_{a 1}}^{\phi_{s t}+\psi_{a 2}}\left(\int_{r}^{\frac{R}{\tan (\beta)} \cdot\left(\phi_{j}-\phi_{e x}\right)}\left(\sin \left(\phi_{j}-\frac{2 \tan \beta}{D} z\right)\right) \mathrm{d} z\right) \mathrm{d} \phi+ \\
+\int_{\phi_{s t}+\psi_{a 2}}^{\phi_{e x}+\psi_{a 1}}\left(\int_{r}^{a}\left(\sin \left(\phi_{j}-\frac{2 \tan \beta}{D} z\right)\right) \mathrm{d} z\right) \mathrm{d} \phi+ \\
+\int_{\phi_{e x}+\psi_{a 1}}^{\phi_{e x}+\psi_{a 2}}\left(\int_{\frac{R}{\tan (\beta)} \cdot\left(\phi_{j}-\phi_{e x}\right)}^{a}\left(\sin \left(\phi_{j}-\frac{2 \tan \beta}{D} z\right)\right) \mathrm{d} z\right) \mathrm{d} \phi
\end{array}\right],
\end{aligned}
$$


and

$$
C_{21}=-\frac{N_{f}}{2 \pi}\left[\begin{array}{l}
\int_{\phi_{s t}+\psi_{a 1}}^{\phi_{s t}+\psi_{a 2}}\left(\int_{r}^{\frac{D}{\tan (\beta)} \cdot\left(\phi_{j}-\phi_{s t}\right)}(\mathrm{d} z)\right) \mathrm{d} \phi+ \\
+\int_{\phi_{s t}+\psi_{a 2}}^{\phi_{e x}+\psi_{a 1}}\left(\int_{r}^{a} \mathrm{~d} z\right) \mathrm{d} \phi+\int_{\phi_{e x+a 1}}^{\phi_{e x}+{ }_{a 2}}\left(\int_{\frac{D}{\tan (\beta)} \cdot\left(\phi_{j}-\phi_{s t}\right)}^{a} \mathrm{~d} z\right) \mathrm{d} \phi
\end{array}\right]
$$

Then, the average cutting forces in part (1) and part (2) of the cutting edge were calculated by (47) and (48):

$$
\begin{aligned}
& \left\{\begin{array}{l}
\bar{F}_{f 1}=\left(C_{1} K_{t c}+C_{3} K_{r c}+C_{5} K_{a c}\right) f_{t}+C_{2} K_{t e}+C_{4} K_{r e}+C_{6} K_{a e} ; \\
\bar{F}_{n 1}=\left(C_{7} K_{t c}+C_{9} K_{r c}+C_{11} K_{a c}\right) f_{t}+C_{8} K_{t e}+C_{10} K_{r e}+C_{12} K_{a e} ; \\
\bar{F}_{a 1}=\left(C_{13} K_{r c}+C_{15} K_{a c}\right) f_{t}+C_{14} K_{r e}+C_{16} K_{a e},
\end{array}\right. \\
& \left\{\begin{array}{l}
\bar{F}_{f 2}=\left(C_{17} K_{t c}+C_{18} K_{r c}\right) f_{t}+C_{19} K_{t e}+C_{20} K_{r e} ; \\
\bar{F}_{n 2}=\left(-C_{18} K_{t c}+C_{17} K_{r c}\right) f_{t}-C_{20} K_{t e}+C_{19} K_{r e} ; \\
\bar{F}_{a 2}=-C_{20} K_{a c} f_{t}+C_{21} K_{a e} .
\end{array}\right.
\end{aligned}
$$

So, the average cutting forces in the entire of one cutting edge were calculated by (49):

$$
\left\{\begin{array}{l}
\bar{F}_{f}=\left(\left(C_{1}+C_{17}\right) K_{t c}+\left(C_{3}+C_{18}\right) K_{r c}+C_{5} K_{a c}\right) f_{t}+\left(C_{2}+C_{19}\right) K_{t e}+\left(C_{4}+C_{20}\right) K_{r e}+C_{6} K_{a e} \\
\bar{F}_{n}=\left(\left(C_{7}-C_{18}\right) K_{t c}+\left(C_{9}+C_{17}\right) K_{r c}+C_{11} K_{a c}\right) f_{t}+\left(C_{8}-C_{20}\right) K_{t e}+\left(C_{10}+C_{19}\right) K_{r e}+C_{12} K_{a e} \\
\bar{F}_{a}=\left(C_{13} K_{r c}+\left(C_{15}-C_{20}\right) K_{a c}\right) f_{t}+C_{14} K_{r e}+\left(C_{16}+C_{21}\right) K_{a e}
\end{array}\right.
$$

Besides, the average cutting forces can be regressed by the linear functions of feed per flute as by (50):

$$
\left\{\begin{array}{l}
\bar{F}_{f}=\bar{F}_{f c} \cdot f_{t}+\bar{F}_{f e} \\
\bar{F}_{n}=\bar{F}_{n c} \cdot f_{t}+\bar{F}_{n e} \\
\bar{F}_{a}=\bar{F}_{a c} \cdot f_{t}+\bar{F}_{a e}
\end{array}\right.
$$

where $\bar{F}_{f c}, \bar{F}_{f e}, \bar{F}_{n c}, \bar{F}_{n e}, \bar{F}_{a c}, \bar{F}_{a e}$ are the linear regression constants that were determined by analyzing relationship of the experimental data of average cutting forces and feed per flute. From the (49) and (50), the relationship of the linear regression constants $\left(\bar{F}_{f c}, \bar{F}_{f e}, \bar{F}_{n c}, \bar{F}_{n e}, \bar{F}_{a c}, \bar{F}_{a e}\right)$, the setting constants $\left(C_{i}\right)$, and the cutting force coefficients were presented by (51):

$$
\left\{\begin{array}{l}
\bar{F}_{f c}=\left(\left(C_{1}+C_{17}\right) K_{t c}+\left(C_{3}+C_{18}\right) K_{r c}+C_{5} K_{a c}\right) ; \\
\bar{F}_{f e}=\left(C_{2}+C_{19}\right) K_{t e}+\left(C_{4}+C_{20}\right) K_{r e}+C_{6} K_{a e} ; \\
\bar{F}_{n c}=\left(\left(C_{7}-C_{18}\right) K_{t c}+\left(C_{9}+C_{17}\right) K_{r c}+C_{11} K_{a c}\right) \\
\bar{F}_{n e}=\left(C_{8}+C_{20}\right) K_{t e}+\left(C_{10}+C_{19}\right) K_{r e}+C_{12} K_{a e} ; \\
\bar{F}_{a c}=\left(C_{13} K_{r c}+\left(C_{15}-C_{20}\right) K_{a c}\right) \\
\bar{F}_{a e}=C_{14} K_{r e}+\left(C_{16}+C_{21}\right) K_{a e} .
\end{array}\right.
$$


Finally, the cutting force coefficients were calculated by (52):

$$
\begin{aligned}
& k_{a c}=\frac{\bar{F}_{a c}\left(\begin{array}{c}
\left(C_{3}+C_{18}\right)\left(C_{7}-C_{18}\right)\left(C_{15}-C_{20}\right)- \\
-\left(C_{1}+C_{17}\right)\left(C_{9}+C_{17}\right)\left(C_{15}-C_{20}\right)
\end{array}\right)-\left(C_{7}-C_{18}\right) C_{13}\left(C_{15}-C_{20}\right) \bar{F}_{f c}+\left(C_{1}+C_{17}\right) C_{13}\left(C_{15}-C_{20}\right) \bar{F}_{n c}}{\left(C_{3}+C_{18}\right)\left(C_{7}-C_{18}\right)\left(C_{15}-C_{20}\right)^{2}-C_{5}\left(C_{7}-C_{18}\right) C_{13}\left(C_{15}-C_{20}\right)-\left(C_{1}+C_{17}\right)\left(C_{9}+C_{17}\right)\left(C_{15}-C_{20}\right)^{2}+\left(C_{1}+C_{17}\right) C_{11} C_{13}\left(C_{15}-C_{20}\right)} ; \\
& k_{a e}=\frac{\bar{F}_{a e}\left(\begin{array}{l}
\left(C_{4}+C_{20}\right)\left(C_{8}+C_{20}\right)\left(C_{16}+C_{21}\right)- \\
-\left(C_{2}+C_{19}\right)\left(C_{10}+C_{19}\right)\left(C_{16}+C_{21}\right)
\end{array}\right)-\left(C_{8}+C_{20}\right) C_{14}\left(C_{16}+C_{21}\right) \bar{F}_{f e}+\left(C_{2}+C_{19}\right) C_{14}\left(C_{16}+C_{21}\right) \bar{F}_{n e}}{\left(C_{4}+C_{20}\right)\left(C_{8}+C_{20}\right)\left(C_{16}+C_{21}\right)^{2}-C_{6}\left(C_{8}+C_{20}\right) C_{14}\left(C_{16}+C_{21}\right)-\left(C_{2}+C_{19}\right)\left(C_{10}+C_{19}\right)\left(C_{16}+C_{21}\right)^{2}+\left(C_{2}+C_{19}\right) C_{12} C_{14}\left(C_{16}+C_{21}\right)} ; \\
& \left\{k_{r c}=\frac{\bar{F}_{a c}\left(\begin{array}{c}
\left(C_{1}+C_{17}\right) C_{11}- \\
-C_{5}\left(C_{7}-C_{18}\right)
\end{array}\right)+\left(C_{7}-C_{18}\right) C_{15} \bar{F}_{f c}-\left(C_{1}+C_{17}\right)\left(C_{15}-C_{20}\right) \bar{F}_{n c}}{\left(C_{3}+C_{18}\right) C_{7}\left(C_{15}-C_{20}\right)-C_{5}\left(C_{7}-C_{18}\right) C_{13}-\left(C_{1}+C_{17}\right)\left(C_{9}+C_{17}\right)\left(C_{15}-C_{20}\right)+\left(C_{1}+C_{17}\right) C_{11} C_{13}} ;\right. \\
& \bar{F}_{a e}\left(\begin{array}{c}
\left(C_{2}+C_{19}\right) C_{12}- \\
-C_{6}\left(C_{8}+C_{20}\right)
\end{array}\right)+\left(C_{8}+C_{20}\right)\left(C_{16}+C_{21}\right) \bar{F}_{f e}-\left(C_{2}+C_{19}\right)\left(C_{16}+C_{21}\right) \bar{F}_{n e} \\
& k_{r e}=\frac{\left(C_{4}+C_{20}\right)\left(C_{8}+C_{20}\right)\left(C_{16}+C_{21}\right)-C_{6}\left(C_{8}+C_{20}\right) C_{14}-\left(C_{2}+C_{19}\right)\left(C_{10}+C_{19}\right)\left(C_{16}+C_{21}\right)+\left(C_{2}+C_{19}\right) C_{12} C_{14}}{;} \\
& \begin{array}{l}
k_{t c}=\frac{\bar{F}_{f c}-\left(C_{3}+C_{18}\right) \bar{F}_{r c}-C_{5} \bar{F}_{a c}}{\left(C_{1}+C_{17}\right)} \\
k_{t e}=\frac{\bar{F}_{f e}-\left(C_{3}+C_{18}\right) \bar{F}_{r e}-C_{6} \bar{F}_{a e}}{\left(C_{2}+C_{19}\right)}
\end{array}
\end{aligned}
$$

The experimental process was performed to verify the relationship of average cutting force and feed per flute as presented by (50), to determine the cutting force coefficients as presented by (52), and to verify the cutting force models in face milling process as presented by (21) and (22). The cutting tests were performed in face milling processes of $\mathrm{C} 45$ steel using the cutter with parallelogram insert. The compositions of C45 steel are listed in Table $\mathbf{1 .}$

\section{Table 1}

Chemical compositions of C45 Steel

\begin{tabular}{|c|c|c|c|c|c|}
\hline Properties & Elastic modulus & Poisson's ratio & Shear modulus & Density & Hardness \\
\hline Unit & $\mathrm{GPa}$ & - & Gpa & $\mathrm{kg} / \mathrm{m}^{3}$ & HRC \\
\hline Value & 200 & 0.3 & 80 & 7800 & 23 \\
\hline
\end{tabular}

\begin{tabular}{ccccccccccc}
\hline Elements & $\mathbf{C}$ & $\mathbf{S i}$ & $\mathbf{M n}$ & $\mathbf{C r}$ & $\mathbf{N i}$ & $\mathbf{M o}$ & $\mathbf{V}$ & $\mathbf{T i}$ & $\mathbf{B}$ & $\mathbf{C u}$ \\
\hline Composite (\%) & 0.44 & 0.23 & 0.65 & 0.15 & 0.15 & 0.04 & 0.01 & 0.001 & 0.0004 & 0.21
\end{tabular}

The main mechanical and physical properties of C45 steel are presented in Table 2. The workpiece dimensions (length $\times$ width $\times$ height) were $45 \times 45 \times 45 \mathrm{~mm}$ as shown in Fig. 6 .

\section{Table 2}

Mechanical and Physical properties of C45 Steel

Fig. 6. Experimental workpiece and cutter insert: $a$-Workpiece; $b$ - Cutter insert

A series of face milling experiments were performed. The cutter was chosen as follows: Face milling cutter with diameter $D=20 \mathrm{~mm}$. The parallelogram cutter insert (R390-11 T3 08M-PM 1025) 
was using with helix angle $\beta=30^{\circ}$, rake angle $\alpha_{r}=5^{\circ}$, and insert nose radius $r=0.4 \mathrm{~mm}$ as shown in Fig. 6. The number of insert $N_{f}$ was 1 insert.

Milling machine was used for experimental method was five-axis CNC machine center (Model: DMU 50 ECOLINE) with SINUMERIK S840DSB control system as shown in Fig. 7. This CNC machine has the specifications as follow: $X / Y / Z$ axis stroke: $500 / 450 / 400 \mathrm{~mm}, X / Y / Z$ axis rapid: $30 / 30 / 30 \mathrm{~m} / \mathrm{min}$, and the maximum spindle speed: $14000 \mathrm{rpm}$.

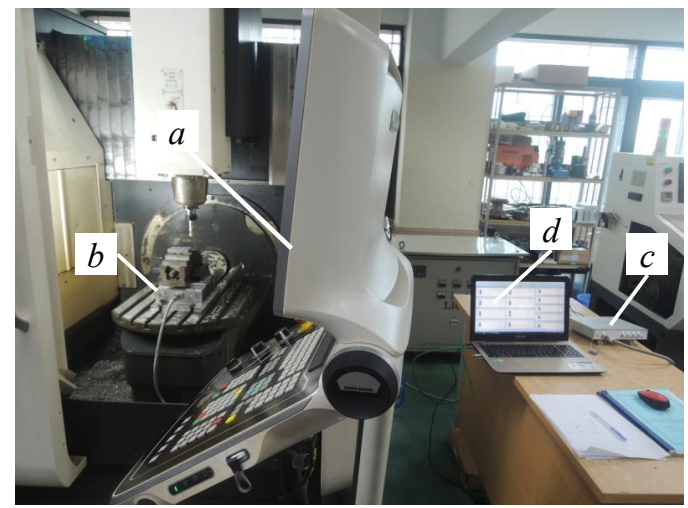

Fig. 7. CNC machine and cutting force measurement system:

$a$ - CNC machine; $b$ - Dynamometer; $c$ - Processing System; $d$ - Software and PC

A dynamometer system was used to measure the cutting forces in experimental work. The KISTLER dynamometer system with dynamometer: Type 9139AA and the auxiliary equipments. The detail of this system is illustrated in Fig. 7. In the setting process, the dynamometer was fixed on the table of CNC machine. The dynamometer was connected to the computer through the processing system that including the data processing box (3160-B-042) and the multi-channel charge amplifier by using connecting cable. The DynoWare software was used to store and analyze the experimental data of cutting forces.

The cutting tests include two sets as listed in Table 3. Set 1 was performed to verify the relationship of the average cutting force and feed per flute and determine the cutting force coefficients. In these cases, the cutting tests were carried out in slot milling (facing). The cutting conditions were selected at the stable cutting conditions with small of cutting depth and small of spindle speed to decrease the influence of chatter and vibrations [9, 14].

Table 3

Experimental plan

\begin{tabular}{ccccc}
\hline Run. No & $a(\mathbf{m m})$ & $b(\mathbf{m m})$ & $f t(\mathbf{m m} /$ flute) & $n(\mathbf{r p m})$ \\
\hline & Set 1: Verification of average force model and determination of cutting force coefficients & \\
\hline 1 & 0.5 & 20 & 0.1 & 750 \\
2 & 0.5 & 20 & 0.15 & 750 \\
3 & 0.5 & 20 & 0.2 & 750 \\
4 & 0.5 & 20 & 0.25 & 750 \\
5 & 0.5 & 20 & 0.3 & 750 \\
\hline 6 & & Set 2: Verification of dynamic cutting force model & 1000 \\
7 & 0.2 & 20 & 0.4 & 850
\end{tabular}

At the constant depth of cut and constant spindle speed, the cutting tests were repeated with variations of feed per flute. Set 2 was performed to verify the cutting force models. In these cases, the cutting tests were selected with different cutting conditions. The cutting parameters of all cutting tests were listed in Table 3. 


\section{Results and Discussions}

3. 1. Verification of Average Cutting Force Model and Determination of Cutting Force Coefficients in Face Milling Process

The cutting tests were performed at stable cutting conditions with small of cutting depth and small of spindle speed (set 1) as listed in Table 3. For each experiment, the average cutting force in feed, normal, and axial directions were calculated from the measured data of cutting forces. The relationship of the average cutting force and the feed per flute was estimated and illustrated in Fig. 8. In this figure, all the absolute values of average cutting forces increase with the increasing of feed per flute. This obtained result was quite the same with the results when using the flat-end mill as presented in reference [9] and when milling using ball-end mill tool as presented in reference [10].

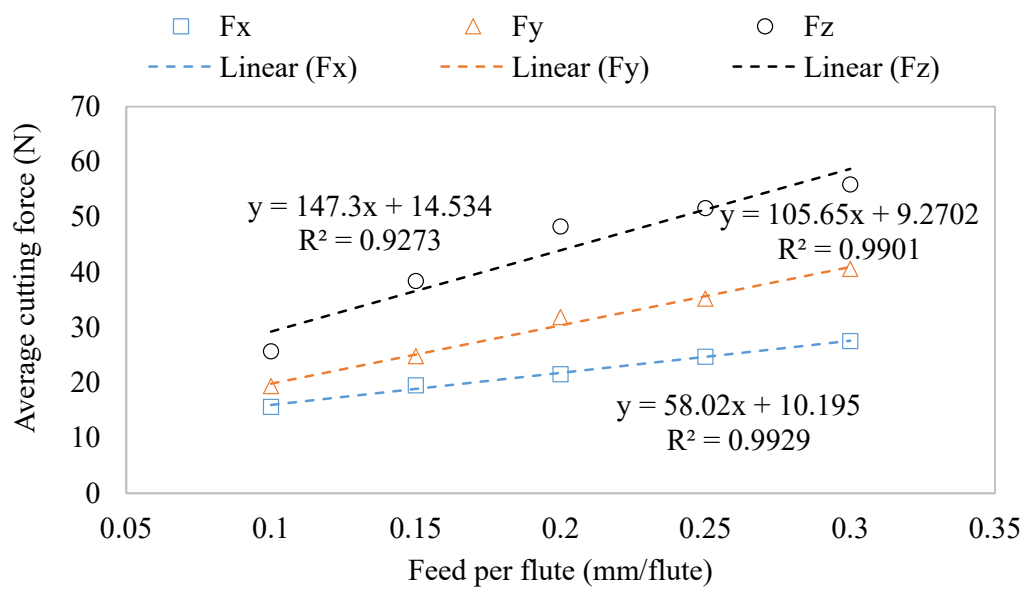

Fig. 8. Relationship of average cutting forces and feed per flute

The verified results show that the theoretical and experimental results have a good agreement. So, in face milling process using parallelogram cutter insert, the relationship of average cutting forces and feed per flute can be expressed as a linear function. In this study, the measured average cutting force can be expressed by the linear function of feed per flute with the large determination coefficients $R^{2}$ (from $92.73 \%$ to $99.29 \%$ ) as shown in Fig. 8. So, the average cutting force models were successfully verified by the analyzed of the relationship of average cutting forces and feed per flute. The analyzed result again proves that in face milling processes, the average cutting force can be modeled as a linear function of feed per flute not only with a specific pair of cutting tool and workpiece as mentioned in some previous studies [10, 14] but also different pairs of cutting tool and workpiece. Applying (52), the measured average cutting forces were used to determine all six cutting force coefficients as listed in Table 4.

Table 4

Calculated values of the cutting force coefficients

\begin{tabular}{cc}
\hline Shearing force coefficient $\left(\mathbf{N} / \mathbf{m m}^{2}\right)$ & Edge force coefficient $(\mathbf{N} / \mathbf{m m})$ \\
\hline$K_{t c}=1592.8$ & $K_{t e}=1.2028$ \\
$K_{r c}=859.9$ & $K_{r e}=12.8051$ \\
$K_{a c}=-1855.2$ & $K_{a e}=-37.4348$
\end{tabular}

The obtained results from Table 4 showed that the absolute values of shear force coefficients $\left(K_{t c}, K_{r c}, K_{a c}\right)$ are often much larger than those ones of edge force coefficients $\left(K_{t e}, K_{r e}, K_{a e}\right)$. So, by analyzing the average cutting force data and the relationship of average cutting forces and feed per flute, all six cutting force coefficient components can be determined in face milling processes. 


\section{2. Verification of the proposed cutting force models in face milling process using} parallelogram cutter insert

Using the obtained cutting force coefficient as stored in Table 4, the cutting forces were predicted and compared with the measured cutting forces. The predicted cutting forces and measured cutting forces were compared and shown from Fig. 9 to Fig. 11 for different cutting parameters (set 1 and set 2). The results from these figures showed that, for different cutting parameters, the predicted cutting forces were quite close to the measured cutting forces both the shape and the amplitude values. In the verifying cutting tests, exiting some different points between predicted cutting forces and measured forces (highlighted by circle and error in Fig. 9 to Fig. 11).

Fig. 9 showed the compared results of predicted and measured cutting force for normal cutting condition (medium depth of cut: $0.5 \mathrm{~mm}$, medium feed per flute: $0.1 \mathrm{~mm} /$ flute, medium small spindle speed: $750 \mathrm{rpm}$ ), therefore, the predicted cutting forces were very close to the measured cutting forces. This issue can be explained that with the normal cutting condition and with small spindle speed, the vibrations were quite mall, then the influence of vibrations on the cutting forces are also small, so the predicted cutting forces were very close to the measured cutting forces.

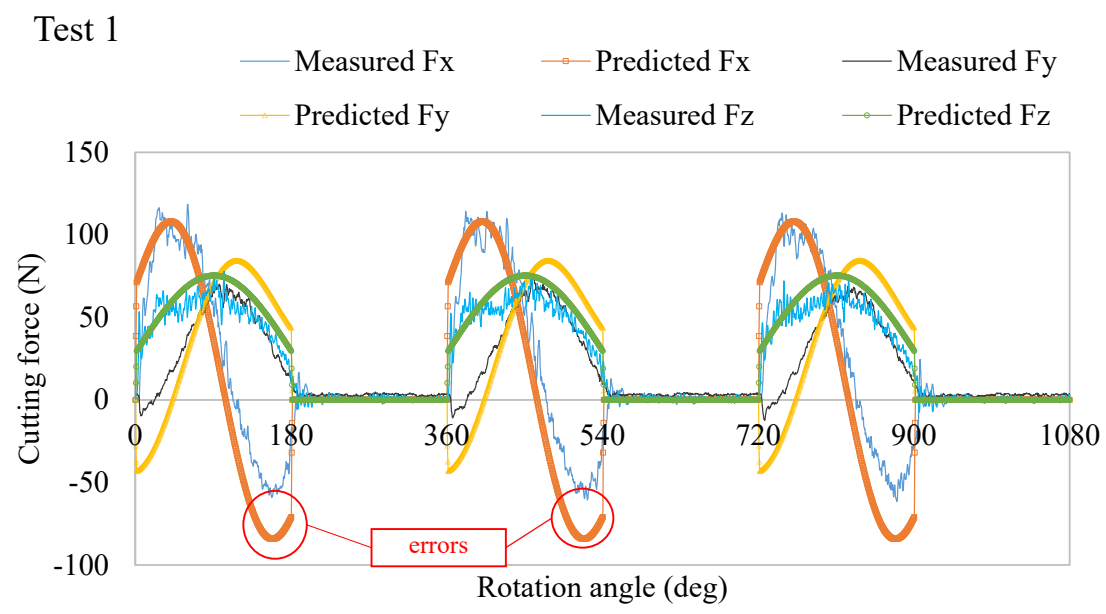

Fig. 9. Comparison of predicted and measured cutting forces of Test 1

These evaluated results of cutting forces were the same the results in Fig. 11 (test 7). Despite the large feed per flute, the depth of cut is a medium value, and the spindle speed is a small value, so this cutting condition is the normal cutting conditions. Therefore, the influence of vibrations on cutting forces was quite small, and so the difference of predicted and measured cutting forces was quite small.

Fig. 10 showed the compared results of predicted and measured cutting force for small depth of cut $(0.5 \mathrm{~mm})$, large feed per flute $(0.5 \mathrm{~mm} /$ flute), and larger spindle speed $(750 \mathrm{rpm})$, the predicted cutting forces were more different to the measured cutting forces. This issue can be explained that with the larger spindle speed, the vibrations were bigger, then the influence of vibrations on the cutting forces was also larger, so the predicted cutting forces were different to the measured cutting forces.

Although there are still differences between the predicted and measured cutting forces, in general, these differences are quite small. Therefore, the predicted results from research model agree satisfactorily with experimental results. So, from all above analyzed results, the cutting force models and cutting force coefficient models in this study can be used to determine the cutting force coefficients and predict the cutting force in face milling process using parallelogram cutter insert.

The reasons for the above differences can be from the vibrations, the chatter, the cutter runout, the deflection, the inconstancy of cutting depth, the unstable of workpiece hardness, the temperature, the friction, and so on. Although, the effect of factors (vibration, noise, temperature, etc.) is limited in the experiments, but in fact, this effect still exists in the experiment processes. Integration of other parameters such as vibrations, cutter run-out, deflection, temperature, etc into cutting force models will be the research direction in the future to overcome the limitations of this study. 


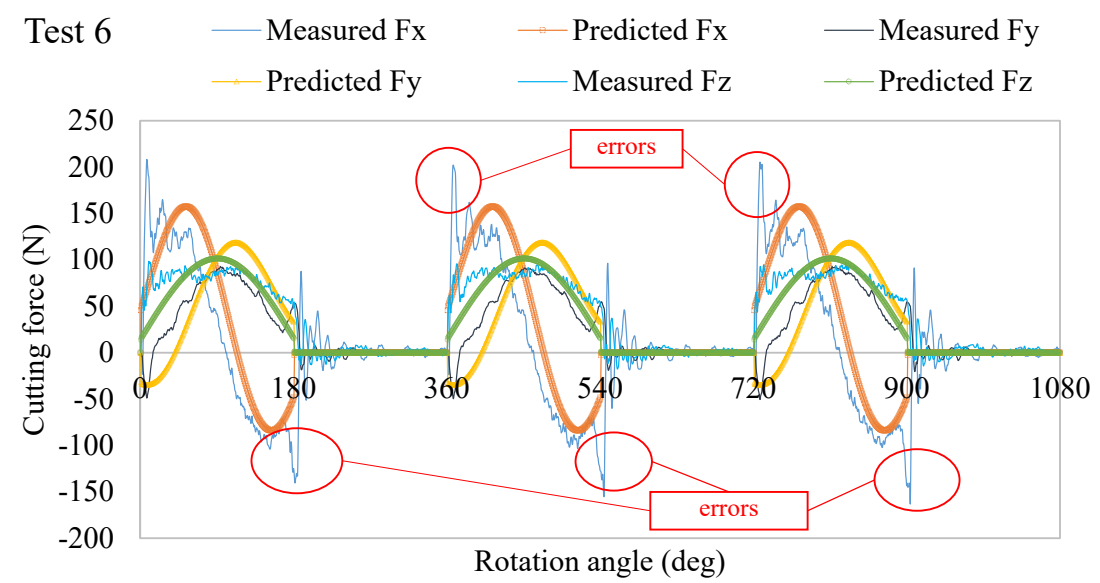

Fig. 10. Comparison of predicted and measured cutting forces of Test 6

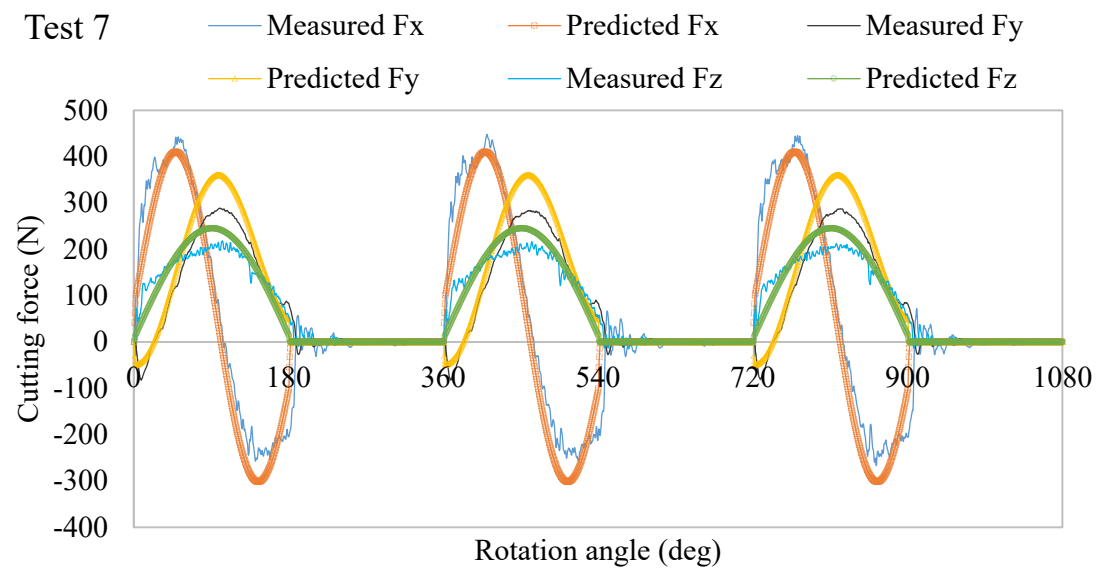

Fig. 11. Comparison of predicted and measured cutting forces of Test 7

\section{Conclusions}

By analyzing the theoretical face milling process using a parallelogram cutter insert, the average cutting forces can be modeled as the linear function of feed per flute.

By the experimental method, the average cutting forces in three directions $(x, y, z)$ can also be modeled as the linear function of feed per flute with the large determination coefficients $R^{2}$ (from $92.73 \%$ to $99.29 \%$ ). The average cutting force models were successfully verified by the analysis of the relationship of average cutting forces and feed per flute.

By analyzing the average cutting force data and the relationship of average cutting forces and feed for flute. Which, the absolute values of shear force coefficients $\left(K_{t c}, K_{r c}, K_{a c}\right)$ are often much larger than those ones of edge force coefficients $\left(K_{t e}, K_{r e}, K_{a e}\right)$.

The predicted results from the research model agree satisfactorily with experimental results. So, from all the above-analyzed results, the cutting force models and cutting force coefficient models in this study can be used to determine the cutting force coefficients and predict the cutting force in the face milling process using a parallelogram cutter insert.

The reasons for the differences between predicted and measured cutting forces can be from the vibrations, the chatter, the cutter run-out, the deflection, the inconstancy of cutting depth, the unstable workpiece hardness, the temperature, the friction, and so on. Investigation of these parameters on the cutting forces and values of cutting force coefficients will the next research directions of this study.

\section{Acknowledgements}

The authors appreciate the support from the research project 02-2020-RD/HĐ-ĐHCN. Thanks also extend to the support from the Faculty of Mechanical Engineering, Hanoi University of Industry, Vietnam. 


\section{References}

[1] Hoang, D. T., Nguyen, N.-T., Tran, Q. D., Nguyen, T. V. (2019). Cutting Forces and Surface Roughness in Face-Milling of SKD61 Hard Steel. Strojniški Vestnik - Journal of Mechanical Engineering, 65 (6), 375-385. doi: https://doi.org/10.5545/ sv-jme.2019.6057

[2] Salguero, J., Batista, M., Calamaz, M., Girot, F., Marcos, M. (2013). Cutting Forces Parametric Model for the Dry High Speed Contour Milling of Aerospace Aluminium Alloys. Procedia Engineering, 63, 735-742. doi: https://doi.org/10.1016/ j.proeng.2013.08.215

[3] Irgolic, T., Cus, F., Paulic, M., Balic, J. (2014). Prediction of Cutting Forces with Neural Network by Milling Functionally Graded Material. Procedia Engineering, 69, 804-813. doi: https://doi.org/10.1016/j.proeng.2014.03.057

[4] Budak, E. (2006). Analytical models for high performance milling. Part I: Cutting forces, structural deformations and tolerance integrity. International Journal of Machine Tools and Manufacture, 46 (12-13), 1478-1488. doi: https://doi.org/10.1016/ j.ijmachtools.2005.09.009

[5] Wan, M., Lu, M.-S., Zhang, W.-H., Yang, Y. (2012). A new ternary-mechanism model for the prediction of cutting forces in flat end milling. International Journal of Machine Tools and Manufacture, 57, 34-45. doi: https://doi.org/10.1016/j.ijmachtools.2012.02.003

[6] Perez, H., Diez, E., Marquez, J. J., Vizan, A. (2013). An enhanced method for cutting force estimation in peripheral milling. The International Journal of Advanced Manufacturing Technology, 69 (5-8), 1731-1741. doi: https://oi.org/10.1007/ s00170-013-5153-0

[7] Wang, B., Hao, H., Wang, M., Hou, J., Feng, Y. (2013). Identification of instantaneous cutting force coefficients using surface error. The International Journal of Advanced Manufacturing Technology, 68 (1-4), 701-709. doi: https://oi.org/10.1007/ s00170-013-4792-5

[8] Wang, M., Gao, L., Zheng, Y. (2014). An examination of the fundamental mechanics of cutting force coefficients. International Journal of Machine Tools and Manufacture, 78, 1-7. doi: https://doi.org/10.1016/j.ijmachtools.2013.10.008

[9] Kao, Y.-C., Nguyen, N.-T., Chen, M.-S., Su, S.-T. (2014). A prediction method of cutting force coefficients with helix angle of flat-end cutter and its application in a virtual three-axis milling simulation system. The International Journal of Advanced Manufacturing Technology, 77 (9-12), 1793-1809. doi: https://doi.org/10.1007/s00170-014-6550-8

[10] Kao, Y.-C., Nguyen, N.-T., Chen, M.-S., Huang, S.-C. (2015). A combination method of the theory and experiment in determination of cutting force coefficients in ball-end mill processes. Journal of Computational Design and Engineering, 2 (4), 233-247. doi: https://doi.org/10.1016/j.jcde.2015.06.005

[11] Gao, G., Wu, B., Zhang, D., Luo, M. (2013). Mechanistic identification of cutting force coefficients in bull-nose milling process. Chinese Journal of Aeronautics, 26 (3), 823-830. doi: https://doi.org/10.1016/j.cja.2013.04.007

[12] Euan, I. G., Ozturk, E., Sims, N. D. (2013). Modeling Static and Dynamic Cutting Forces and Vibrations for Inserted Ceramic Milling Tools. Procedia CIRP, 8, 564-569. doi: https://doi.org/10.1016/j.procir.2013.06.151

[13] Engin, S., Altintas, Y. (2001). Mechanics and dynamics of general milling cutters.: Part II: inserted cutters. International Journal of Machine Tools and Manufacture, 41 (15), 2213-2231, doi: https://doi.org/10.1016/s0890-6955(01)00046-3

[14] Agarwal, A., Desai, K. A. (2020). Importance of bottom and flank edges in force models for flat-end milling operation. The International Journal of Advanced Manufacturing Technology, 107 (3-4), 1437-1449. doi: https://doi.org/10.1007/s00170-020-05111-5

How to cite: Nguyen, N.-T. (2021). A development method of cutting force coefficients in face milling process using parallelogram insert. EUREKA: Physics and Engineering, 5, 36-52. doi: https://doi.org/10.21303/2461-4262.2021.001890 